\title{
On the Analysis of Numerical Methods for Nonstandard Volterra Integral Equation
}

\author{
H. S. Mamba and M. Khumalo \\ Department of Pure and Applied Mathematics, University of Johannesburg, P.O. Box 524, Auckland Park 2006, South Africa \\ Correspondence should be addressed to H. S. Mamba; hlukaphi@gmail.com
}

Received 16 September 2014; Revised 27 November 2014; Accepted 27 November 2014; Published 15 December 2014

Academic Editor: Józef Banaś

Copyright ( $) 2014$ H. S. Mamba and M. Khumalo. This is an open access article distributed under the Creative Commons Attribution License, which permits unrestricted use, distribution, and reproduction in any medium, provided the original work is properly cited.

\begin{abstract}
We consider the numerical solutions of a class of nonlinear (nonstandard) Volterra integral equation. We prove the existence and uniqueness of the one point collocation solutions and the solution by the repeated trapezoidal rule for the nonlinear Volterra integral equation. We analyze the convergence of the collocation methods and the repeated trapezoidal rule. Numerical experiments are used to illustrate theoretical results.
\end{abstract}

\section{Introduction}

In this paper we consider the nonlinear (nonstandard) Volterra integral equation:

$$
u(t)=\sum_{j=1}^{2} b_{j}\left(g_{j}(t)+\int_{0}^{t} k_{j}(t, s) u(s) d s\right)^{j}, \quad t \in[0, T],
$$

where $b_{j} \in \mathbb{R}$ and $g_{j}, k_{j}$ are continuous functions. Some examples of the nonlinear VIE (1) arise from nonlinear ordinary differential equations used to represent conservative systems. In [1] we provided sufficient conditions for the existence and uniqueness of the solution to (1). We also approximated the solutions for (1) using collocation methods, the repeated trapezoidal rule, and repeated Simpson's rule.

Collocation solutions for Volterra integral equations have been the subject of several work over the years. Brunner [2] studied the existence and uniqueness of the collocation solution for linear Volterra integral equations of the second kind and showed that the methods yield global convergence of order $m$. In [3] nonlinear Volterra integral equations with multiple proportional delays were studied; they provided sufficient conditions for the existence and uniqueness of the analytic and collocation solution. Moreover, [3] proved that the collocation methods yield global convergence of order $m$. Other authors such as [4-6] also analysed the convergence of collocation methods for Volterra integral equations with different types of kernels. In this work we study the conditions for the existence and uniqueness of the numerical solutions of (1) and perform convergence analysis for the collocation methods and repeated trapezoidal rule.

\section{Existence and Uniqueness of the Numerical Solution}

Consider the nonlinear VIE

$$
v(t)=b\left(g(t)+\int_{0}^{t} k(t, s) v(s) d s\right)^{2},
$$

where $k \in C(D),(D:=(t, s): 0 \leq s \leq t \leq T)$, and $g \in C(I)$. We prove a theorem analogous to the one in [3], establishing the existence and uniqueness of the collocation solution for (2). The collocation solution to (2) is given as

$$
\begin{aligned}
V_{n, 1}=b( & g\left(t_{n, 1}\right)+F_{n}\left(t_{n, 1}\right) \\
& \left.+h\left(\int_{0}^{c_{1}} k\left(t_{n, 1}, t_{n}+s h\right) d s\right) V_{n, 1}\right)^{2},
\end{aligned}
$$

where

$$
F_{n}\left(t_{n, 1}\right)=h \sum_{i=0}^{n-1}\left(\int_{0}^{1} k\left(t_{n, 1}, t_{i}+s h\right) d s\right) V_{i, 1} .
$$


Let

$$
B_{n}=\int_{0}^{c_{1}} k\left(t_{n, 1}, t_{n}+s h\right) d s,
$$

$g=g\left(t_{n, 1}\right)$, and $F=F_{n}\left(t_{n, 1}\right)$; then

$$
\begin{gathered}
V_{n, 1}=b\left(g^{2}+2 g F+F^{2}+2 g h B_{n} V_{n, 1}\right. \\
\left.+2 h F B_{n} V_{n, 1}+h^{2} B_{n}^{2} V_{n, 1}^{2}\right) \\
{\left[1-h\left(2 b g B_{n}+2 b F B_{n}+h B_{n}^{2} V_{n, 1}\right)\right] V_{n, 1}=b(g+F)^{2} .}
\end{gathered}
$$

To solve (7) we use an iterative procedure; thus we rewrite (7) in the form

$$
\left[1-h\left(2 b g B_{n}+2 b F B_{n}+h B_{n}^{2} V_{n, 1}^{(s)}\right)\right] V_{n, 1}^{(s+1)}=b(g+F)^{2},
$$

where $s=1,2, \ldots$ Let

$$
\wp^{(s)}=\left(2 b g B_{n}+2 b F B_{n}+h B_{n}^{2} V_{n, 1}^{(s)}\right) .
$$

Then (8) can be rewritten as

$$
\left[1-h \mathfrak{\wp}^{(s)}\right] V_{n, 1}^{(s+1)}=b(g+F)^{2} .
$$

Theorem 1. Assume that the given functions $g$ and $k$ in the nonlinear VIE (2) are continuous in their respective domains $I$ and $D$. Then there exists a constant $\bar{h}>0$, for any uniform mesh $I_{h}$ with $h<\bar{h}$, such that (3) defines a unique collocation solution $v_{h}$ that belongs to the piecewise constant polynomial $S_{0}^{(-1)}\left(I_{h}\right)$, for (2).

Proof. Since the kernel $k$ in (2) is continuous on $D$, then $\wp^{(1)}$ in (10) is bounded. Whenever $h\left|\wp^{(1)}\right|<1,1-h \wp^{(1)} \neq 0$. Hence, for $s=1$ in (10), $V_{n, 1}^{(2)}$ exists and is bounded.

For some $s>1$ assume that $V_{n, 1}^{(s)}$ is bounded. Then, arguing as above, $V_{n, 1}^{(s+1)}$ exists and is bounded if $h\left|\wp^{(s)}\right|<1$.

The above holds if there is an $\bar{h}>0$ such that for a uniform mesh $I_{h}$ with $h<\bar{h}$, the condition $h\left|\wp^{(s)}\right|<1$ holds for all $s \geq 1$. Hence there exists a unique solution $V_{n, 1}^{(s+1)}$ for (3).

Corollary 2. Under the same conditions as in Theorem 1,

$$
\begin{aligned}
v\left(t_{n}\right)=b\left(g\left(t_{n}\right)+\frac{h}{2} k\left(t_{n}, t_{0}\right) v\left(t_{0}\right)\right. \\
\left.+h \sum_{i=1}^{n-1} k\left(t_{n}, t_{i}\right) v\left(t_{i}\right)+\frac{h}{2} k\left(t_{n}, t_{n}\right) v\left(t_{n}\right)\right)^{2}
\end{aligned}
$$

defines a unique solution to (2) by the repeated trapezoidal rule.
Proof. Expanding (11) we get

$$
\begin{gathered}
v_{n}-h\left(b g_{n} k_{n, n}+b \frac{h}{2} k_{n, 0} v_{0} k_{n, n}\right. \\
\left.+b h \sum_{i=1}^{n-1} k_{n, i} v_{i} k_{n, n}+b \frac{h}{2}\left(k_{n, n}\right)^{2} v_{n}\right) v_{n} \\
=b\left[g_{n}^{2}+g_{n} h k_{n, 0} v_{0}+\left(\frac{h}{2} k_{n, 0} v_{0}\right)^{2}\right. \\
+\left(\sum_{i=1}^{n-1} k_{n, i} v_{i}\right)^{2}+2 g_{n} h \sum_{i=1}^{n-1} k_{n, i} v_{i} \\
\left.+h^{2} k_{n, 0} v_{0} \sum_{i=1}^{n-1} k_{n, i} v_{i}\right],
\end{gathered}
$$

where $v_{n}=v\left(t_{n}\right), g_{n}=g\left(t_{n}\right)$, and $k_{n, i}=k\left(t_{n}, t_{i}\right)$ for $i=0, \ldots$, $n$. Let

$$
\wp^{(s)}=\left(b g_{n} k_{n, n}+b \frac{h}{2} k_{n, 0} v_{0} k_{n, n}+b h \sum_{i=1}^{n-1} k_{n, i} v_{i} k_{n, n}\right.
$$

$$
\left.+b \frac{h}{2}\left(k_{n, n}\right)^{2} v_{n}^{(s)}\right)
$$

then we have

$$
\begin{aligned}
& {\left[1-h \wp^{(s)}\right] v_{n}^{(s+1)}} \\
& =b\left[g_{n}^{2}+g_{n} h k_{n, 0} v_{0}+\left(\frac{h}{2} k_{n, 0} v_{0}\right)^{2}+\left(\sum_{i=1}^{n-1} k_{n, i} v_{i}\right)^{2}\right. \\
& \left.\quad+2 g_{n} h \sum_{i=1}^{n-1} k_{n, i} v_{i}+h^{2} k_{n, 0} v_{0} \sum_{i=1}^{n-1} k_{n, i} v_{i}\right] .
\end{aligned}
$$

The result follows from Theorem 1 by taking

$$
v_{n}^{(s+1)}=V_{n, 1}^{(s+1)} .
$$

Corollary 3. Applying collocation methods or the repeated trapezoidal rule results in a unique solution for (1).

Proof. Existence and uniqueness for the case $b_{1}=0$, $b_{2} \neq 0$ follows from Theorem 1 and Corollary 2. For the case $b_{1} \neq 0, b_{2}=0$ existence and uniqueness for the collocation solution is established in [2]. For the case $b_{1} \neq 0$, $b_{2}=0$ the existence and uniqueness of the solution by the repeated trapezoidal rule follows from continuity of $g$ and $k$. Combining these two cases establishes the existence and uniqueness of the collocation solution and the solution by the repeated trapezoidal rule for (1). 


\section{Convergence for the Numerical Methods}

3.1. Global Convergence for the Collocation Methods. The global convergence for the case where $b_{2}=0$ in (1) has been analysed (see [2]); thus we will study the case where $b_{1}=0$ and where both $b_{1}$ and $b_{2}$ are nonzero. We use a procedure analogous to the one used in [3] to analyse the global convergence of the collocation solution $v_{h} \in S_{0}^{-1}\left(Z_{N}\right)$, with $Z_{N}:=\left\{t_{0}, \ldots, t_{N}\right\}$.

Theorem 4. Assume that $k \in C(D), g \in C(I)$, and $v_{h} \in$ $S_{0}^{-1}\left(Z_{N}\right)$, defined by (3), are the collocation solution for (2).

Then for all sufficiently small $h>0$, we have

$$
\begin{gathered}
\left\|v_{h}-v\right\|_{\infty} \leq C\left(\left\|\left(1-\mathscr{P}_{h}\right) b g^{2}\right\|_{\infty}+\left\|\left(\kappa-\alpha \mathscr{P}_{h}\right) \beta v\right\|_{\infty}\right), \\
\left\|v_{h}-v\right\|_{\infty}:=\sup _{t \in I}\left|v_{h}(t)-v(t)\right| \leq C h\|v\|_{\infty},
\end{gathered}
$$

where $\mathscr{P}_{h}$ is the Lagrange interpolation operator corresponding to the collocation parameter $c_{1}$ and the constant $C$ does not depend on $h$.

Proof. Define $\beta v$ as follows:

$$
\beta v=\int_{0}^{t} k(t, s) v(s) d s
$$

then the operator formulations for the VIE (2) and its collocation equation are given by

$$
\begin{gathered}
v=b(g+\beta v)^{2}, \\
v_{h}=b \mathscr{P}_{h}\left(g+\beta v_{h}\right)^{2} .
\end{gathered}
$$

Based on the solvability of the VIE and its collocation equation we implement an iterative procedure and obtain

$$
\begin{gathered}
v^{(s+1)}=(1-\kappa \beta)^{-1}{b g^{2},}^{(s+1)}=\left(1-\alpha \beta \mathscr{P}_{h}\right)^{-1} \mathscr{P}_{h} b g^{2},
\end{gathered}
$$

where $\kappa=2 b g \beta+b \beta^{2} v^{(s)}$ and $\alpha=2 b g \beta \mathscr{P}_{h}+b \beta^{2} \mathscr{P}_{h} v_{h}^{(s)}$.

Then the error between $v_{h}$ and $v$ can be written as

$$
\begin{aligned}
e_{h}:= & v_{h}-v \\
= & \left(1-\alpha \mathscr{P}_{h} \beta\right)^{-1} \mathscr{P}_{h} b g^{2}-(1-\kappa \beta)^{-1} b g^{2} \\
= & (1-\kappa \beta)^{-1} \mathscr{P}_{h} b g^{2}+\left(1-\alpha \mathscr{P}_{h} \beta\right)^{-1} \mathscr{P}_{h} b g^{2} \\
& -(1-\kappa \beta)^{-1}\left(b g^{2}-\mathscr{P}_{h} b g^{2}\right) \\
= & \left(1-\alpha \mathscr{P}_{h} \beta\right)^{-1}\left(\kappa \beta-\alpha \mathscr{P}_{h} \beta\right)(1-\kappa \beta)^{-1} \mathscr{P}_{h} b g^{2} \\
& +(1-\kappa \beta)^{-1}\left(b g^{2}-\mathscr{P}_{h} b g^{2}\right) \\
= & \left(1-\alpha \mathscr{P}_{h} \beta\right)^{-1}\left(\kappa \beta-\alpha \mathscr{P}_{h} \beta\right)(1-\kappa \beta)^{-1}\left(\mathscr{P}_{h} b g^{2}-b g^{2}\right) \\
& +\left(1-\alpha \mathscr{P}_{h} \beta\right)^{-1}\left(\kappa \beta-\alpha \mathscr{P}_{h} \beta\right)(1-\kappa \beta)^{-1} b g^{2} \\
& +(1-\kappa \beta)^{-1}\left(1-\mathscr{P}_{h}\right) b g^{2}
\end{aligned}
$$

$$
\begin{aligned}
= & \left(1-\alpha \mathscr{P}_{h} \beta\right)^{-1}\left(\kappa \beta-\alpha \mathscr{P}_{h} \beta\right)(1-\kappa \beta)^{-1}\left(\mathscr{P}_{h}-1\right) b g^{2} \\
& +\left(1-\alpha \mathscr{P}_{h} \beta\right)^{-1}\left(\kappa-\alpha \mathscr{P}_{h}\right) \beta v \\
& +(1-\kappa \beta)^{-1}\left(1-\mathscr{P}_{h}\right) b g^{2},
\end{aligned}
$$

which implies that

$$
\left\|v_{h}-v\right\|_{\infty} \leq C\left(\left\|\left(1-\mathscr{P}_{h}\right) b g^{2}\right\|_{\infty}+\left\|\left(\kappa-\alpha \mathscr{P}_{h}\right) \beta v\right\|_{\infty}\right) .
$$

From the error estimates of the interpolation $\mathscr{P}_{h}$, we have

$$
\left\|\left(1-\mathscr{P}_{h}\right) b g^{2}\right\|_{\infty} \leq C h\left\|b g^{2}\right\|_{\infty} \leq C h\|v\|_{\infty},
$$

and with appropriate assumptions on $\alpha$ and $\kappa$,

$$
\left\|\left(\kappa-\alpha \mathscr{P}_{h}\right) \beta v\right\|_{\infty} \leq C h\|\beta v\|_{\infty} \leq C h\|v\|_{\infty},
$$

which leads to

$$
\left\|v_{h}-v\right\| \leq C h\|v\|_{\infty} .
$$

On the other hand, recall that the collocation solution to (1) is given by

$$
u_{h}(t)=\sum_{j=1}^{2} b_{j}\left(g_{j}(t)+\int_{0}^{t} k_{j}(t, s) u(s) d s\right)^{j}, \quad t \in X_{N} .
$$

Corollary 5. If the solution $u_{h} \in S_{0}^{-1}\left(Z_{N}\right)$ defined by (25) is the collocation solution to (1), then, for a sufficiently small $h>0$,

$$
\left\|u_{h}-u\right\|_{\infty}:=\sup _{t \in I}\left|u_{h}(t)-u(t)\right| \leq C h\|u\|_{\infty}
$$

holds for any set $X_{N}$ of collocation point with $0 \leq c_{1} \leq 1$. The constant $C$ depends on the $c_{1}$ but not on $h$.

Proof. From [2] we know that the collocation solution for the VIE,

$$
y(t)=g(t)+\int_{0}^{t} k(t, s) y(s) d s
$$

satisfies

$$
\left\|y_{h}-y\right\|_{\infty}:=\sup _{t \in I}\left|y_{h}(t)-y(t)\right| \leq C h\|y\|_{\infty} .
$$

Using the triangle inequality we have

$$
\begin{aligned}
\left\|u_{h}-u\right\|_{\infty} & =\left\|\left(y_{h}-y\right)+\left(v_{h}-v\right)\right\|_{\infty} \\
& \leq\left\|\left(y_{h}-y\right)\right\|_{\infty}+\left\|\left(v_{h}-v\right)\right\|_{\infty} \\
& \leq C h\|y\|_{\infty}+C h\|v\|_{\infty} \\
& \leq C h\left(\|y\|_{\infty}+\|v\|_{\infty}\right) .
\end{aligned}
$$


3.2. Repeated Trapezoidal Rule. Consider the solution to (2) by repeated trapezoidal rule

$$
v\left(t_{n}\right)=b\left(g\left(t_{n}\right)+h \sum_{i=0}^{n} w_{i} k\left(t_{n}, t_{i}\right) v\left(t_{i}\right)\right)^{2} .
$$

Theorem 6. The approximate method given by (30) is convergent and its order of convergence is at least one.

Proof. Putting $t=t_{n}$ in (2), we have

$$
\begin{aligned}
& \left|\varepsilon_{n}\right|=\left|v_{h}\left(t_{n}\right)-v\left(t_{n}\right)\right| \\
& =\mid b\left[\left(h \sum_{i=0}^{n} 2 g\left(t_{n}\right) w_{i} k\left(t_{n}, t_{i}\right) v\left(t_{i}\right)\right)\right. \\
& -\left(2 g\left(t_{n}\right) \int_{0}^{t_{n}} k\left(t_{n}, s\right) v(s) d s\right) \\
& +\left(h \sum_{i=0}^{n} w_{i} k\left(t_{n}, t_{i}\right) v\left(t_{i}\right)\right)^{2} \\
& \left.-\left(\int_{0}^{t_{n}} k\left(t_{n}, s\right) v(s) d s\right)^{2}\right] \\
& =\mid b\left[\left(h \sum_{i=0}^{n} 2 g\left(t_{n}\right) w_{i} k\left(t_{n}, t_{i}\right) v\left(t_{i}\right)\right)\right. \\
& -\left(2 g\left(t_{n}\right) \int_{0}^{t_{n}} k\left(t_{n}, s\right) v(s) d s\right) \\
& +\left(h \sum_{i=0}^{n} w_{i} k\left(t_{n}, t_{i}\right) v\left(t_{i}\right)-\int_{0}^{t_{n}} k\left(t_{n}, s\right) v(s) d s\right) \\
& \cdot\left(h \sum_{i=0}^{n} w_{i} k\left(t_{n}, t_{i}\right) v\left(t_{i}\right)\right. \\
& \left.\left.+\int_{0}^{t_{n}} k\left(t_{n}, s\right) v(s) d s\right)\right] \\
& =\mid b\left[\left(h \sum_{i=0}^{n} 2 g\left(t_{n}\right) w_{i} k\left(t_{n}, t_{i}\right) v\left(t_{i}\right)\right)\right. \\
& -\left(2 g\left(t_{n}\right) \int_{0}^{t_{n}} k\left(t_{n}, s\right) v(s) d s\right) \\
& +\left(h \sum_{i=0}^{n} w_{i} k\left(t_{n}, t_{i}\right) v\left(t_{i}\right)-\int_{0}^{t_{n}} k\left(t_{n}, s\right) v(s) d s\right) \\
& \cdot\left(h \sum_{i=0}^{n} w_{i} k\left(t_{n}, t_{i}\right) v\left(t_{i}\right)\right. \\
& -\int_{0}^{t_{n}} k\left(t_{n}, s\right) v(s) d s \\
& \left.\left.+2 \int_{0}^{t_{n}} k\left(t_{n}, s\right) v(s) d s\right)\right] \mid
\end{aligned}
$$

Using the Lipschitz condition, (31) can be written as

$$
\begin{aligned}
\left|\varepsilon_{n}\right| \leq & h A_{1} \sum_{i=0}^{n}\left|\varepsilon_{i}\right|+\left|R_{i, 1}\right| \\
& +\left(h A_{2} \sum_{i=0}^{n}\left|\varepsilon_{i}\right|+\left|R_{i, 1}\right|\right)\left(h A_{2} \sum_{i=0}^{n}\left|\varepsilon_{i}\right|+\left|R_{i, 2}\right|\right),
\end{aligned}
$$

where $R_{i, 1}$ and $R_{i, 2}$ are the errors of the integration rule.

Then, let $R=\max _{i}\left[R_{i, 1}, R_{i, 2}\right]$; hence

$$
\begin{aligned}
\left|\varepsilon_{n}\right| \leq & \frac{h A_{1}}{1-h A_{1}} \sum_{i=0}^{n-1}\left|\varepsilon_{i}\right|+\frac{R}{1-h A_{1}} \\
& +\left(\frac{h A_{2}}{1-h A_{2}} \sum_{i=0}^{n-1}\left|\varepsilon_{i}\right|+\frac{R}{1-h A_{2}}\right) \\
& \cdot\left(\frac{h A_{2}}{1-h A_{2}} \sum_{i=0}^{n-1}\left|\varepsilon_{i}\right|+\frac{R}{1-h A_{2}}\right) .
\end{aligned}
$$

Then we have

$$
\begin{aligned}
\left|\varepsilon_{n}\right| \leq & \frac{1}{1-h A_{1}}\left\{R+h A_{1} \sum_{i=0}^{n-1}\left|\varepsilon_{i}\right|\right\} e^{\left(A_{1} t_{n} /\left(1-A_{1}\right)\right)} \\
& +\left(\frac{1}{1-h A_{2}}\left\{R+h A_{2} \sum_{i=0}^{n-1}\left|\varepsilon_{i}\right|\right\} e^{\left(A_{2} t_{n} /\left(1-A_{2}\right)\right)}\right) \\
& \cdot\left(\frac{1}{1-h A_{2}}\left\{R+h A_{2} \sum_{i=0}^{n-1}\left|\varepsilon_{i}\right|\right\} e^{\left(A_{2} t_{n} /\left(1-A_{2}\right)\right)}\right) .
\end{aligned}
$$

For the functions $k$ and $g$ with at least first-order derivatives, we have $R=O(h)$. Hence we have

$$
\begin{aligned}
\left|\varepsilon_{n}\right| & =O(h)+O(h) O(h) \\
& =O(h)+O\left(h^{2}\right) \\
& =O(h)
\end{aligned}
$$

Corollary 7. The repeated trapezoidal solution for (1), defined by

$$
u\left(t_{n}\right)=\sum_{j=1}^{2} b\left(g\left(t_{n}\right)+h \sum_{i=0}^{n} w_{i} k\left(t_{n}, t_{i}\right) v\left(t_{i}\right)\right)^{2}
$$

is convergent and its order of convergence is at least one.

Proof. Since $\left|\varepsilon_{n}\right|=O(h)$ for the repeated trapezoidal rule when used to solve (2) and (27), it follows that for (1) we have

$$
\begin{aligned}
\left|\varepsilon_{n}\right| & =O(h)+O(h) \\
& =O(h) .
\end{aligned}
$$




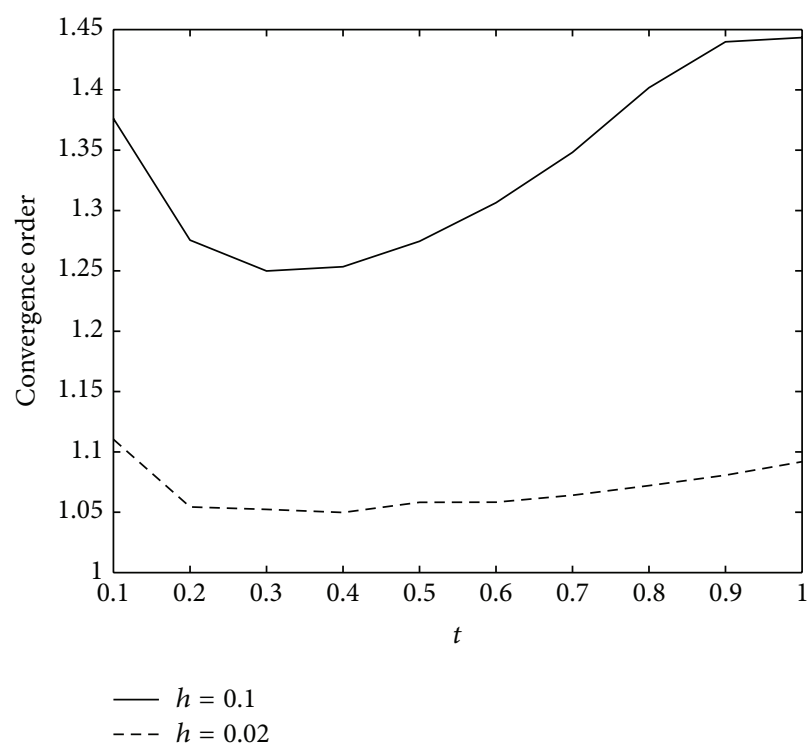

(a) The convergence rates of the implicit Euler

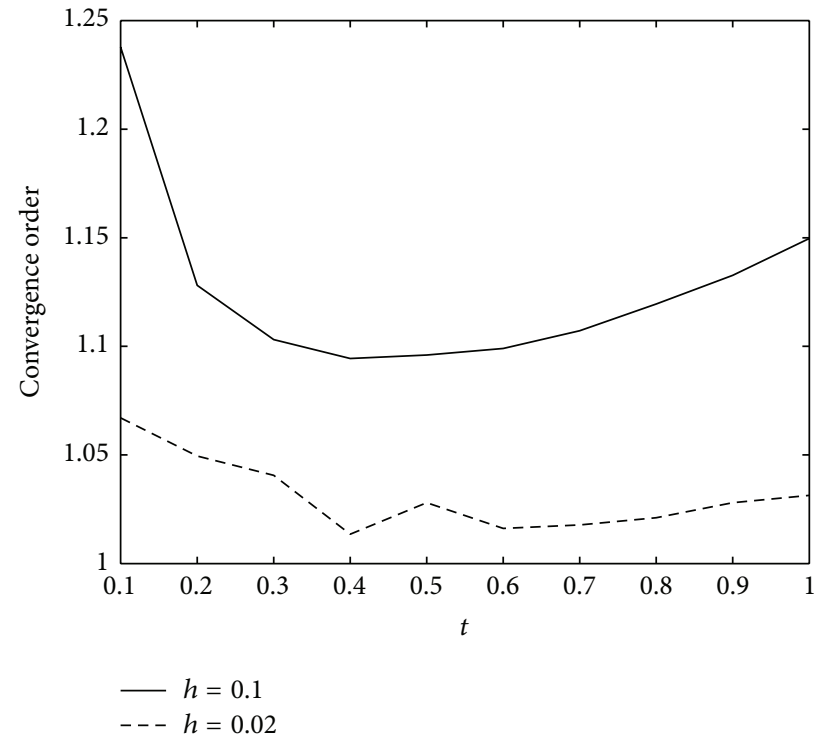

(b) The convergence rates of the implicit midpoint

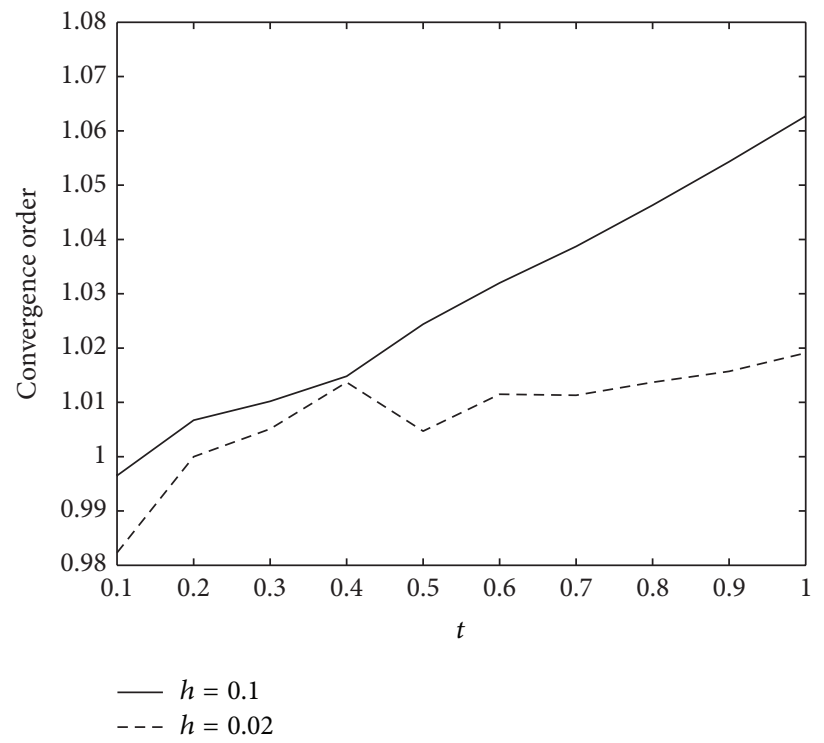

(c) The convergence rates of the repeated trapezoidal rule

FIGURE 1: The convergence rates for (39).

\section{Numerical Results}

In this section we present numerical results obtained from the collocation methods and the repeated trapezoidal rule. To obtain the estimates for the convergence rates we used the following quantity:

$$
p=\frac{\log \left(\left(u^{h / 2}-u^{h}\right) /\left(u^{h / 4}-u^{h / 2}\right)\right)}{\log 2},
$$

where $u^{h}, u^{h / 2}$, and $u^{h / 4}$ denote approximations to $u(t)$ using the step sizes $h, h / 2$, and $h / 4$. The results are shown in Figures 1 and 2, which indicates first-order convergence and this is in agreement with the results of the theorems in Section 3.
The approximations for the convergence rates are done using results from the following examples.

Example 1. Consider

$$
u(t)=2\left(1+\int_{0}^{t}(t-s) u(s) d s\right)^{2} \quad 0 \leq t \leq 1 .
$$

Example 2. Consider

$$
u(t)=\left(1+\int_{0}^{t}(t-s) u(s) d s\right)+\frac{1}{2}\left(1+\int_{0}^{t}(t-s) u(s) d s\right)^{2}
$$




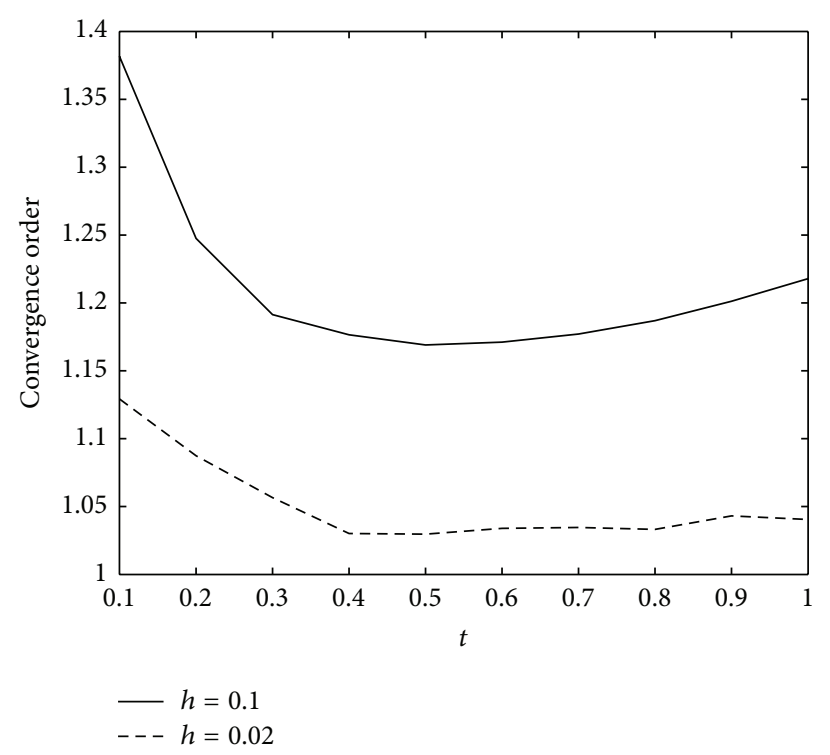

(a) The convergence rates of the implicit Euler

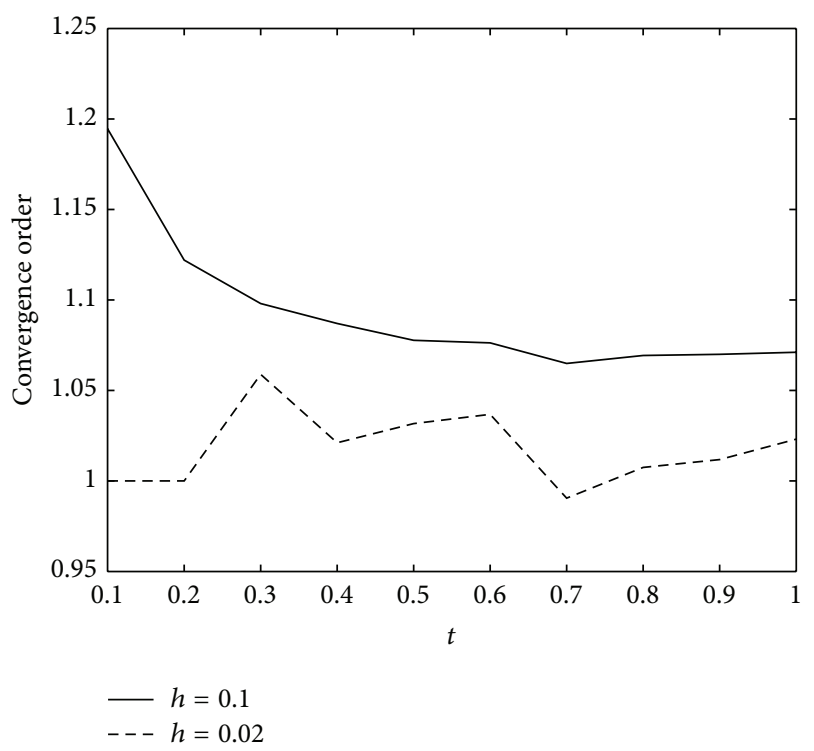

(b) The convergence rates of the implicit midpoint

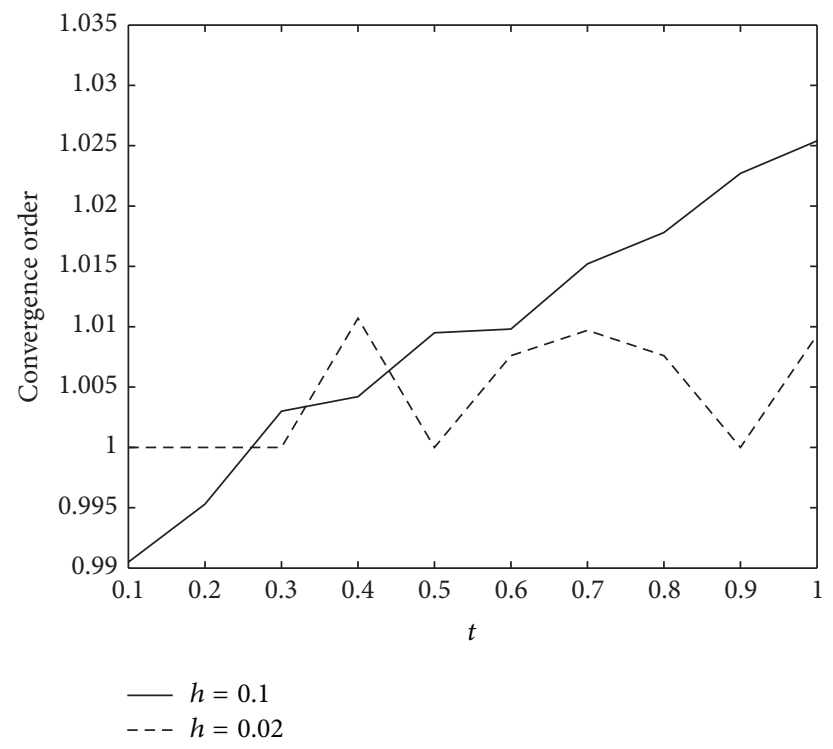

(c) The convergence rates of the repeated trapezoidal rule

FIgURE 2: The convergence rates for (40).

\section{Discussion}

In this work we provided sufficient conditions for the existence and uniqueness of the collocation solution and the solution by the repeated trapezoidal rule for (2). We performed a numerical analysis of the nonlinear (nonstandard) Volterra integral equation (1). In Theorem 4 and Corollary 5 we proved that the collocation methods yield global convergence order of one. We also proved in Section 3.2 that the repeated trapezoidal rule has convergence order of one. Numerical approximations of the convergence orders of the implicit Euler, implicit midpoint, and repeated trapezoidal rule were made and are shown in Section 4. The computed orders of convergence are in agreement with the theoretical results in Section 3.

\section{Conflict of Interests}

The authors declare that there is no conflict of interests in respect of this paper.

\section{References}

[1] H. S. Malindzisa and M. Khumalo, "Numerical solutions of a class of nonlinear Volterra integral equations," Abstract and Applied Analysis, vol. 2014, Article ID 652631, 8 pages, 2014.

[2] H. Brunner, Collocation Methods for Volterra Integral and Related Functional Differential Equations, Cambridge University Press, Cambridge, UK, 2004.

[3] K. Zhang, J. Li, and H. Song, "Collocation methods for nonlinear convolution Volterra integral equations with multiple 
proportional delays," Applied Mathematics and Computation, vol. 218, no. 22, pp. 10848-10860, 2012.

[4] T. Diogo, "Collocation and iterated collocation methods for a class of weakly singular Volterra integral equations," Journal of Computational and Applied Mathematics, vol. 229, no. 2, pp. 363-372, 2009.

[5] T. Diogo and P. Lima, "Collocation solutions of a weakly singular Volterra integral equation," Trends in Applied and Computational Mathematics, vol. 8, no. 2, pp. 229-238, 2007.

[6] V. Horvat, "On collocation methods for Volterra integral equations with delay arguments," Mathematical Communications, vol. 4, no. 1, pp. 93-109, 1999. 


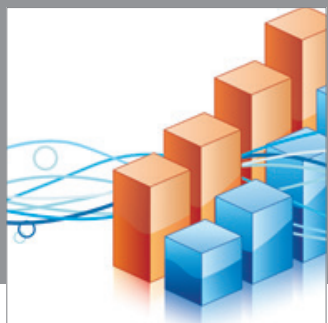

Advances in

Operations Research

mansans

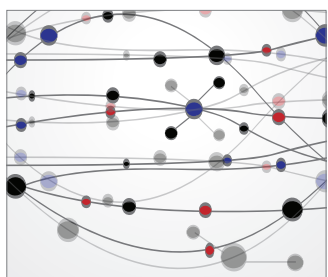

The Scientific World Journal
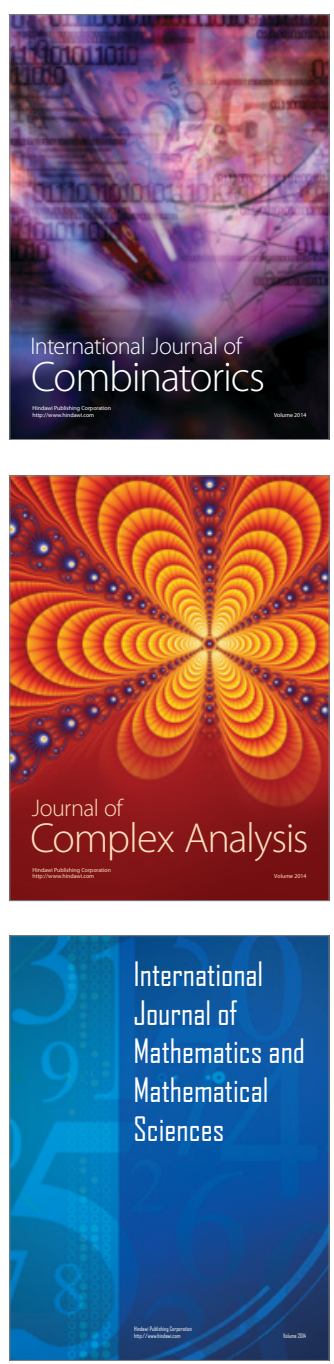
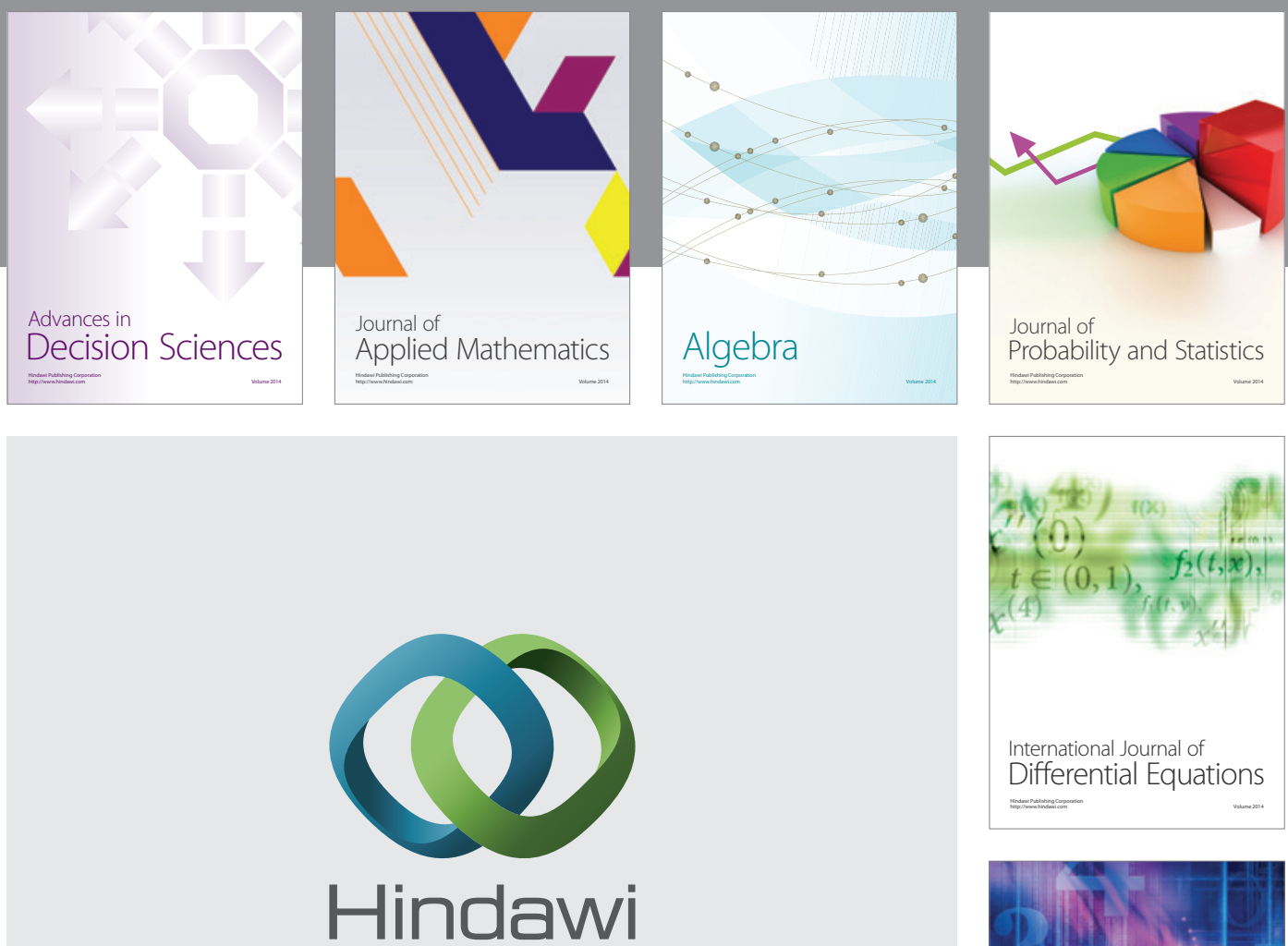

Submit your manuscripts at http://www.hindawi.com
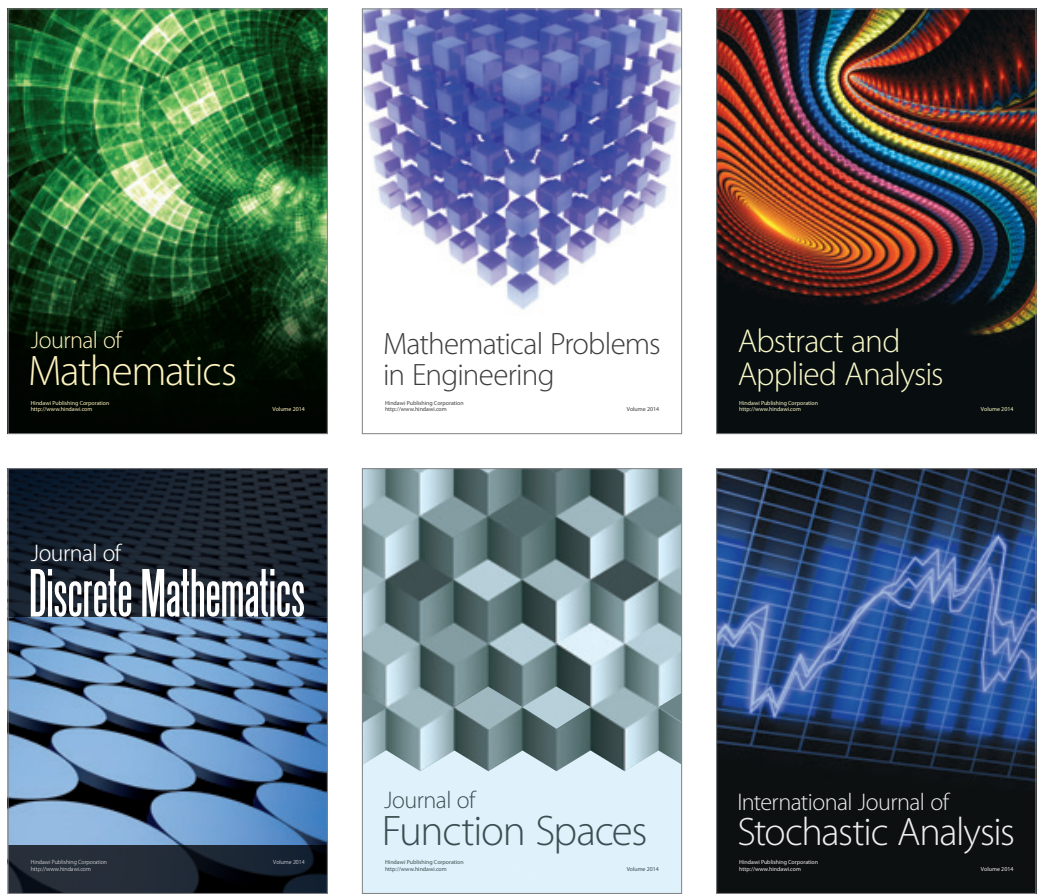

Journal of

Function Spaces

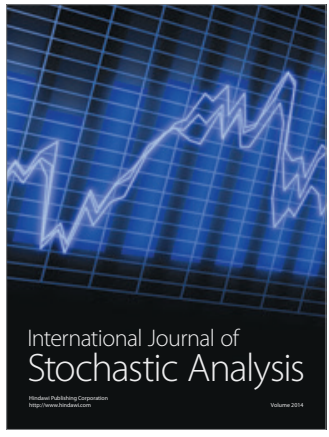

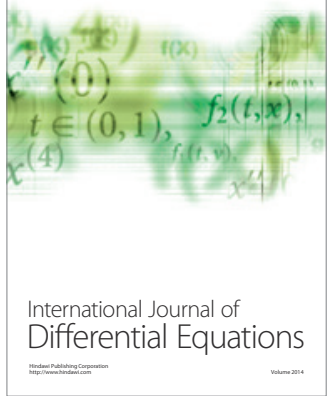
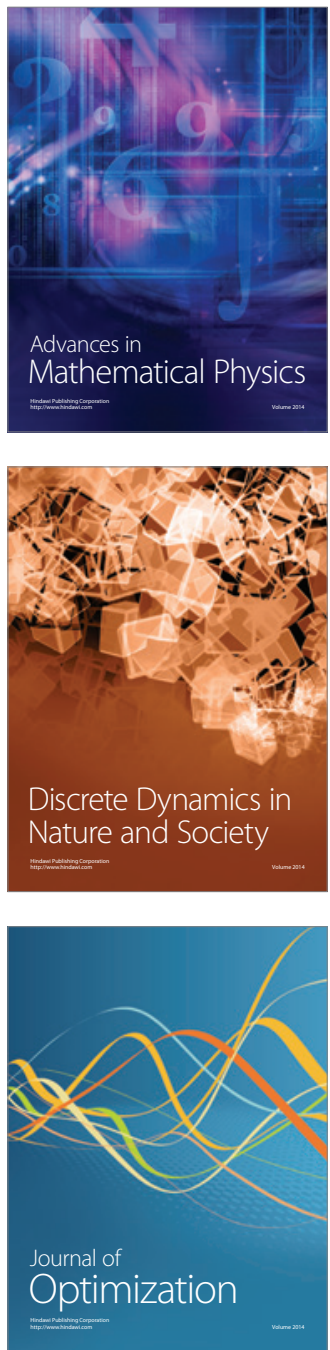